www.jmscr.igmpublication.org

Impact Factor 5.84

Index Copernicus Value: 83.27

ISSN (e)-2347-176x ISSN (p) 2455-0450

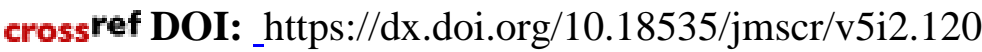

Journal Of Medical Science And Clinical Research

\title{
Changing Trends in the Epidemiology of Pancreatitis in Rural Population: Study at a Tertiary Health Care Centre
}

\author{
Authors \\ Shivani Battin ${ }^{1}$, Dr Ninad Gadekar ${ }^{2}$, Dr Gund ${ }^{3}$ \\ ${ }^{1}$ Intern, Dr. Vitthalrao Vikhe Patil Foundation's Medical College \& Hospital \\ ${ }^{2}$ Assistant Professor, Department of General Surgery \\ ${ }^{3}$ Professor, Department of Preventive and Social Medicine
}

Dr. Vitthalrao Vikhe Patil Foundation's Medical College \& Hospital, Near Govt. Milk Dairy, Vilad Ghat,

Ahmednagar, Maharashtra, India

\begin{abstract}
Introduction: Worldwide, over the last decade, yearly incidence of pancreatitis and related hospitalizations have increased ${ }^{[1]}$. Acute pancreatitis is the most common cause of GI related hospitalizations in the world, with more than 274,000 hospitalizations in $2012^{[1]}$. The yearly incidence of AP in the United States is approximately 70-80 new cases per 100,000 population ${ }^{[2]}$ and has increased over the last decade. Chronic pancreatitis, although lower in incidence, significantly reduces the patients' quality of life. According to the data presented by the Department of the Welfare in $2005^{[3]}$, the rate of patients discharged for chronic pancreatitis is 32.9/100,000 hospitalized patients. True incidence and prevalence of pancreatitis in India is unknown since, it being difficult to establish an accurate diagnosis, a number of cases are misdiagnosed or are not recorded or reported. Nevertheless, in 2004, a high prevalence of chronic pancreatitis in Southern India (114-200/100,000 population) was registered in a study of chronic pancreatitis in the Asia-Pacific region ${ }^{[4]}$.

Material and Method: A retrospective cross-sectional, non-interventional, analytical hospital based study on the proven cases of pancreatitis admitted and treated in Vikhe Patil Hospital, a tertiary level health care centre was conducted. A specific period of 6 months from November 2014 to April 2015 formed the sample under study. The study was started after the approval from the Institutional Ethics Committee by collecting the medical records of the said cases from the Medical Records Department. These records included the history, clinical profile, biochemical and radiological reports, treatments administered, complications and final outcome of the respective cases. The data available in these records was organised and analysed.

Results: Results were analysed based on the following parameters: 1. Demographic data, 2. Etiological factors, 3. Clinical presentation with reference to diagnosis, 4. Investigations, 5. Treatment and final outcome, 6.Complications.

On analysis of the results, this study reaffirms that alcohol is the major factor associated with pancreatitis in the rural population of Maharashtra in India.

Conclusion: The incidence and epidemiology of pancreatitis in Indian settings in under-studied. This report presents the relevant observations noted at a tertiary health care centre comparing them with the available international studies. Here, a hospital based cross-sectional analytical study about the changing epidemiological and clinical pattern of pancreatitis was undertaken. This study comprised of a relatively small sample size which is a great limitation for arriving at definite conclusions; for this, further studies are required both epidemiological and clinical.

Keywords: Changing trends, Pancreatitis, Rural population, Tertiary health care centre.
\end{abstract}




\section{Introduction}

"Acute Pancreatitis is the most terrible of all the calamities that occur in connection with the abdominal viscera. The suddenness of its onset, the illimitable agony which accompanies it, and the mortality attendant upon it, all render it the most formidable of catastrophes!"

-Lord Moynihan (1925) ${ }^{[5]}$

Literally, the meaning of 'Pancreatitis' is inflammation of the pancreas. This inflammatory pancreatic disease is broadly classified based upon the duration of its symptoms and the pathological changes that accompany it into Acute Pancreatitis (AP) and Chronic Pancreatitis (CP). While reversible inflammatory changes are noted in acute pancreatitis, irreversible damage to the pancreas characterises chronic pancreatitis which may range from fibrosis to progressive destruction of both exocrine and endocrine pancreatic tissue. Pancreatitis presents itself in a wide spectrum of clinical presentation from chronic malabsorption to frank acute abdomen.

Mean age of incidence of pancreatitis varies based on the type and aetiology of pancreatitis as well as lifestyle and geographical conditions. It was noted to be within the range of 35-50 years in western countries ${ }^{[6]}$ and around 45-64 years in Italy, with the majority being males ${ }^{[3]}$. In India, mean age of incidence for $\mathrm{CP}$ was found to be around 39.7 years[7]. An increasing trend in the incidence of acute pancreatitis and the total figure of hospitalisations for both acute and chronic pancreatitis has been shown in recent data, especially from predominantly western countries ${ }^{[8-12]}$. This trend has been observed by the practitioners as well; especially in the rural areas.

Being a developing economy, this definitely puts an increased burden on our health care system robbing us of manpower and work force. Besides, the average age of incidence of pancreatitis has also been noted to decrease. Till this date, the exact cause in pathogenesis of pancreatitis is illunderstood. Various theories have been put forth regarding the diverse presentation of pancreatitis.
Numerous factors have been implicated for causation and association of pancreatitis ranging from exogenous elements including lifestyle and behaviour like alcohol ${ }^{[13-15]}$, smoking, tobacco consumption [15,16], nutrition, diet [17,18], environmental toxins, geography [19] to endogenous elements like gallstones $[1,12,20]$, genetics ${ }^{[21]}$, autoimmunity ${ }^{[22]}$,etc.

Further, with the changes in lifestyle, lifestyle diseases are on the rise all over the world. Moreover, with the westernisation of Indian population, the lifestyle of an average Indian has changed drastically in the last few decades. The impact of a changing lifestyle and behaviour, especially in the rural population, is contributing to the modification in the epidemiology of pancreatitis. An example of this is that the alcohol consumption, smoking, etc. in our country has increased ${ }^{[23]}$.

Pancreatitis has a significant rate of mortality and morbidity because of a lack of comprehension of the natural history of occurrence and epidemiology of this disease. Additionally, studies of recent years have confirmed chronic pancreatitis to be a premalignant condition leading to pancreatic carcinoma ${ }^{[24,25]}$.

In spite of pancreatitis being a big drain on our medical and economical resources, shockingly little study has been done of this elusive disease; especially its incidence, occurrence, aetiology and outcome in India. The present data available on the topic is limited, to a great extent, to the western literature. If we have to get the better of this agonising disease, large scale studies for understanding this disease and its prevalence in the Indian scenario are the need of the hour.

This project endeavoured to probe into the obscure areas of epidemiology of pancreatitis, study its changing trends in the rural population and to venture a guess as to the cause of it.

\section{Aims and Objectives}

- To study the changing clinicoepidemiological pattern of pancreatitis in 
the population of rural Maharashtra at a tertiary hospital.

- To study the causative and associative risk factors in the occurrence of pancreatitis.

\section{Material and Methods}

A retrospective cross-sectional, non-interventional, hospital based analytical study based on the proven cases of pancreatitis admitted and treated in a tertiary level health care centre located in rural area was conducted.

All the proven cases of pancreatitis admitted and treated in the centre during a specific period of 6 months from November 2014 to April 2015 formed the sample under study. It was a retrospective, analytical study where the patient data, excluding the personal details, was collected for purely scientific research purpose.

The study was started in the month of July 2015 after the approval from the Institutional Ethics Committee by collecting the medical records of the said cases from the Medical Records Department. These records included the history, clinical profile, biochemical and radiological reports, treatment administered, complications and final outcome of the respective cases. The data available in these records was organised and analysed within a period of two months.Each aspect of the patient's relevant past medical history available in the records was deliberated upon for probable risk factors. In case of patients having recurrent episodes of pancreatitis, their age at the time of the first attack was taken into account for calculating the average age of incidence.

The available reports of biochemical investigations included the routine blood work up like haemoglobin level, Total Leucocyte Count (TLC), blood group, serum liver enzymes, Renal Function Tests (RFTs), serum electrolytes (sodium, potassium and calcium), serum amylase and serum lipase tests. The available radiological data comprised of the Ultrasonography (USG) and CT scan reports. Based on the CT scan reports, the CTSI was calculated according to the Balthazar scoring system ${ }^{[26]}$ and compared to the final outcome of the patient.

The treatment offered, whether conservative or surgical, was noted. The incidence of various complications in acute and chronic pancreatitis was evaluated. The final outcome of the patient, be it death, discharge with follow-up advice or discharge against medical advice was compared to the prognostic variables.

The data of each case was tabulated separately in an Excel master datasheet. The statistical functions like the mean and median were applied. The average ages of incidence, proportions of the various aspects were calculated. Thus, a complete study of each case of pancreatitis occurring in the period of November 2014 to April 2015 was done to ensure that no aspect of the study of epidemiology of pancreatitis was missed and the relevant findings are presented in this report.

\section{Results}

\section{a) Demographic data:}

In a period of 6 months, from November 2014 to April 2015, there were 51 admissions to the hospital with the diagnosis of pancreatitis, of whom, 32 cases were of Acute Pancreatitis (AP) and 19 cases were of Chronic Pancreatitis (CP). Figure 1 shows the distribution of $\mathrm{AP}$ and $\mathrm{CP}$ in the various age groups.

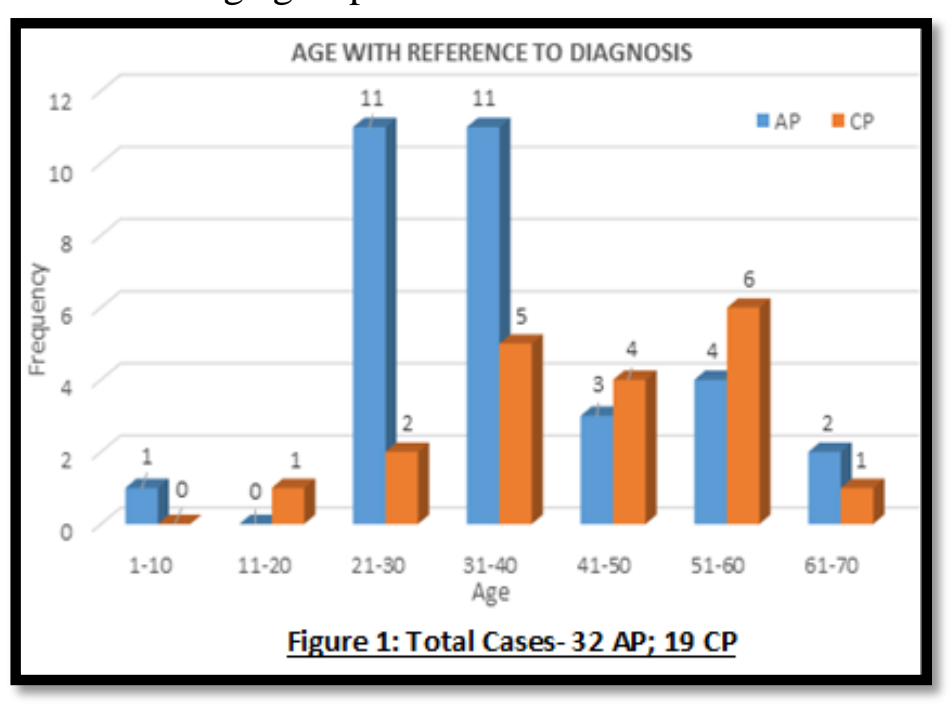

While the ages of the cases ranged from 7-70 years, the mean age of incidence of pancreatitis was 38.39 years. The average age of incidence 
was 38.34 and 38.54 years in case of AP and $\mathrm{CP}$ respectively.

The sex distribution of the cases was as shown in the table 1. Out of the 51 cases, $90.19 \%(n=46)$ were males and $9.80 \%(n=5)$ were females.

\begin{tabular}{|c|c|c|c|}
\hline Sex & $\mathrm{AP}$ & $\mathrm{CP}$ & Total \\
\hline Male & 31 & 15 & 46 \\
\hline Female & 1 & 4 & 5 \\
\hline
\end{tabular}

\section{b) Aetiological Factors:}

Figure 2 illustrates the presence of some of the aetiological factors in the sample under study. Alcohol contributed the most to the aetiology of pancreatitis forming $33 \%$ of all the etiological factors studied here. It was closely followed by tobacco consumption which contributed $26 \%$. Consumption of tobacco was in two forms; viz., smoking of beedis and tobacco chewing. Gallstones were responsible for $18 \%$ of all the cases of pancreatitis; 14 cases gave a positive past history of jaundice or of being diagnosed with gallstones in the past. However, only eight of these cases had icterus on clinical examination. Of these 14 cases, six were diagnosed to be suffering from $\mathrm{AP}$ and the remaining eight from $\mathrm{CP}$. A relatively high contribution was of an iatrogenic factor, namely, Endoscopic Retrograde Cholangio-Pancreatography (ERCP). History of trauma to the abdomen was present in $3 \%$ of the cases. However, in the remaining $11 \%$ of the cases, no evident aetiological factor was found as a probable cause of pancreatitis.

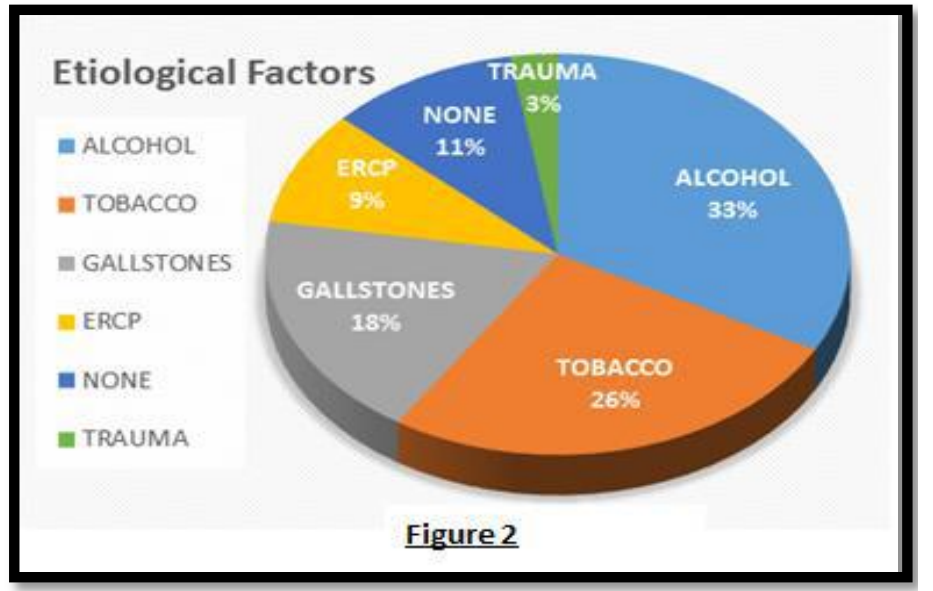

c) Clinical Presentation With Reference To Diagnosis:

Figure 3 displays the extent of the various clinical features the cases presented with. Of the various symptoms the cases presented with, the most common symptom was pain. Abdominal pain was present in all the cases of both, acute as well as chronic pancreatitis.

Presence of fever was another common symptom. 25 cases gave a history of fever, of which, 16 were diagnosed as AP and nine as CP. However, only in 10 cases, it was supported by raised temperature in the records. While 22 of the total 51 cases $(43.13 \%)$ gave a history of nausea, 25 cases gave a positive history of episodes of vomiting. Both, nausea and vomiting were more often associated with AP rather than CP. On the other hand, anorexia, a symptom presented in 20 cases, was relatively more prevalent in $\mathrm{CP}$ $(47.36 \%)$ as compared to AP $(34.37 \%)$. Icterus was present in $29.4 \%(n=15)$ cases, most of which $(n=9)$, were diagnosed to be cases of CP and the remaining ( $n=6)$ as cases of AP. Of these 15 cases having icterus, only five cases had a past history of jaundice or gallstones.

Tenderness of abdomen was the most commonly presenting sign present in $92.15 \%(n=47)$ of the cases of which $61.7 \%$ were AP and $38.29 \% \mathrm{CP}$. Another common presentation was distension of abdomen, reported in $47 \%(n=24)$ cases, of which, only 13 cases had ascites.

While abdominal rigidity was present in $29.4 \%$ $(\mathrm{n}=15)$ cases, $31.3 \%(\mathrm{n}=16)$ cases had guarding of abdomen. $84.61 \%$ of the cases who had both guarding and rigidity $(\mathrm{n}=13)$ were found to be suffering from AP. Like anorexia, a palpable mass in abdomen is another feature more specific to $\mathrm{CP}$ than to AP. In six cases, it was due to liver enlargement, five of which were cases of CP. There was a palpable mass in epigastrium in nine cases (which, on further investigations, mostly turned out to be a pseudocyst). $25.49 \%$ cases gave a history of altered bowel habits. Seven cases suffered from constipation and had hard stools while two had diarrhoea. The remaining four had 


\section{JMSCR Vol||05||Issue||02||Pages 17951-17964||February}

greyish-black coloured stools (one AP and three CP cases). $13.72 \%(n=7)$ cases confirmed having Diabetes Mellitus (DM); three and four of AP and $\mathrm{CP}$ respectively. While this was the first such episode in 34 cases, 17 cases gave a history of recurrent episodes of pancreatitis; of which, nine were cases of AP and eight of CP. On examination, while 14 cases had a blood pressure above the normal $120 / 80 \mathrm{mmHg}$, one case each of $\mathrm{AP}$ and $\mathrm{CP}$ were found to be hypotensive. $21.5 \%$ $(n=11)$ cases had tachycardia, 10 of which were diagnosed to have AP.

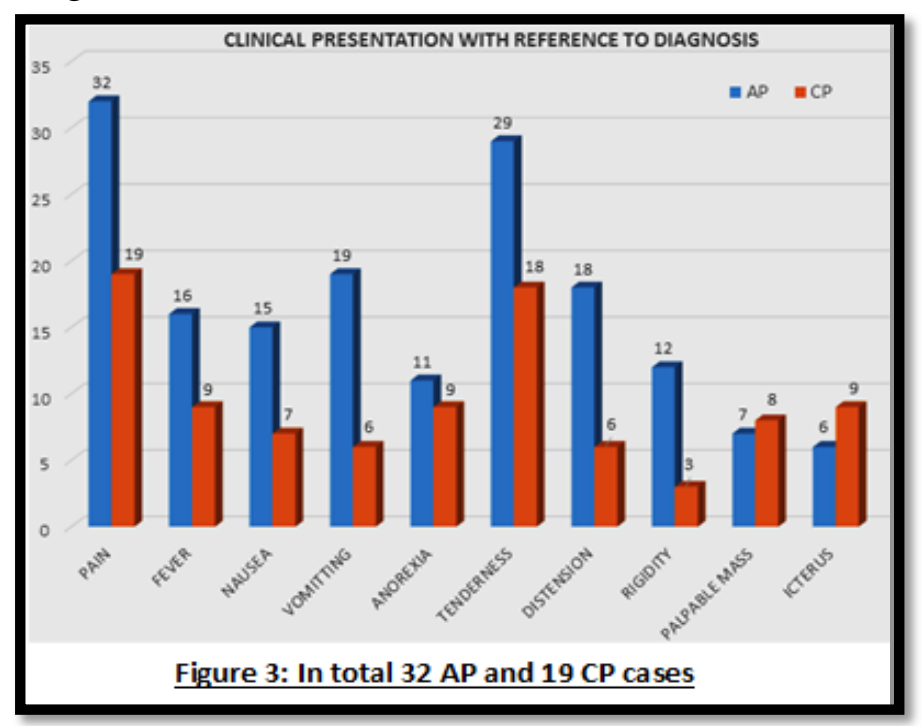

\section{d) Investigations:}

The results laboratory investigations of the cases are shown in figure 4. On blood examination, haemoglobin level of all the cases under study was found to be below the accepted normal range (13.3-16.2 for males and 12-15 for females) except for one case of AP, where it was normal.

Raised WBC count featured in $74.50 \% \quad(n=38)$ cases, of which, 27 cases were of AP and 11 of CP.

Reports of liver enzymes, Aspartate Aminotransferase (AST), Alanine Aminotransferase (ALT) and Alkaline Phosphatase showed derangements. AST was raised in $56.25 \%$ of AP and $63.15 \%$ of CP cases; ALT was raised in $56.25 \%$ and $57.89 \%$ of $\mathrm{AP}$ and $\mathrm{CP}$ cases respectively while alkaline phosphatase showed the maximum rise i.e., in 93.75\% AP and $84.21 \% \mathrm{CP}$ cases. While some cases $(46.87 \%$ AP and $42.10 \%$ CP) showed raised creatinine, almost all the cases $(90.62 \%$ AP and $100 \%$ CP) had a blood urea level above normal.

Serum amylase estimation was done in all the cases while serum lipase levels were recorded in five cases only. Levels of Serum amylase was raised to more than thrice the normal levels in $81.25 \%$ of the AP and $52.63 \%$ of the CP cases. In all the cases where the serum lipase was estimated (four cases of AP and one case of $\mathrm{CP}$ ), it was found to be raised to more than thrice the normal levels.

Serum calcium estimation revealed, hypocalcemia in six AP and two CP cases.

Blood group data of 37 cases was available and its distribution was as shown in figure 5. The most common was blood group B+ve (51.35\%), followed by $\mathrm{O}+\mathrm{ve}(21.62 \%)$. Other blood groups found were $\mathrm{AB}+\mathrm{ve}(13.51 \%), \mathrm{A}+\mathrm{ve}(10.81 \%)$ and B-ve $(2.7 \%)$.

Electrolyte imbalance was also a common finding with 32 cases having hyponatremia (17 AP and 15 $\mathrm{CP}$ ) and two cases showing hypernatremia; both being AP. Reports of seven AP and eight CP cases showed hypokalemia while hyperkalemia was present in six AP and three CP cases.
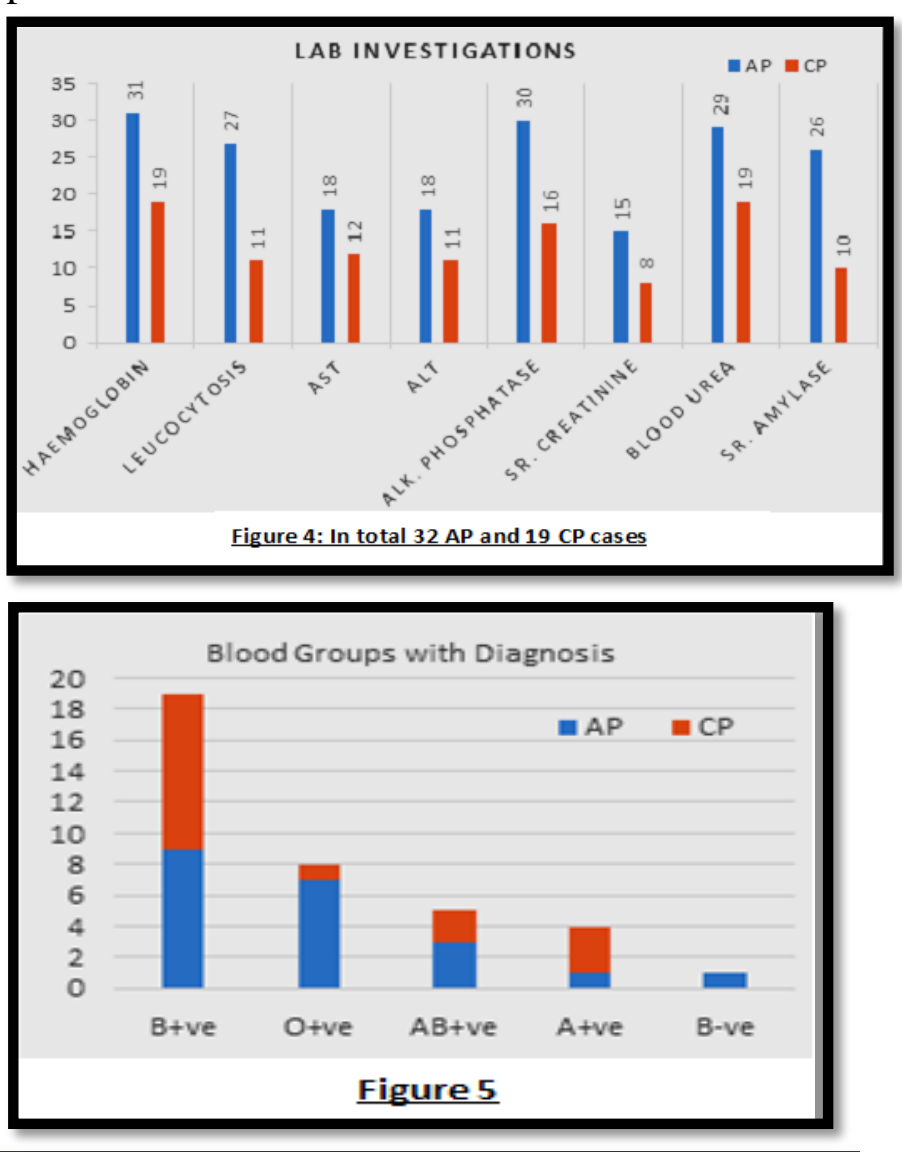
The most common findings in USG reports, as shown in table 2 , were heterogeneous pancreatic appearance, hypoechoic areas in pancreatic mass, cystic or atrophic changes, bulky pancreas, calcifications in pancreatic mass, pancreatic duct calculi or dilated pancreatic duct or some abnormal mass in the pancreatic parenchyma.

Based on CT scan findings, CTSI (CT Severity Index) score was calculated based on the changes in pancreatic mass and Pancreatic Necrosis (according to the Balthazar Grading System). Accordingly, it was observed that, higher the CTSI score more was the mortality. Figure 6 illustrates the CTSI score observed in the cases which is compared to the observed mortality.

\begin{tabular}{|l|l|l|}
\hline USG Changes & AP & CP \\
\hline Heterogeneous & 17 & 8 \\
\hline Hypoechoic & 5 & 5 \\
\hline Cystic & 8 & 3 \\
\hline Bulky & 9 & 0 \\
\hline Calcifications & 1 & 4 \\
\hline Dilated pancreatic duct & 1 & 3 \\
\hline Pancreatic duct calculi & 0 & 4 \\
\hline Gallstones & 2 & 0 \\
\hline Atrophic changes & 0 & 4 \\
\hline Mass Table 2 & 0 & 2 \\
\hline \multicolumn{2}{|c|}{} \\
\hline
\end{tabular}

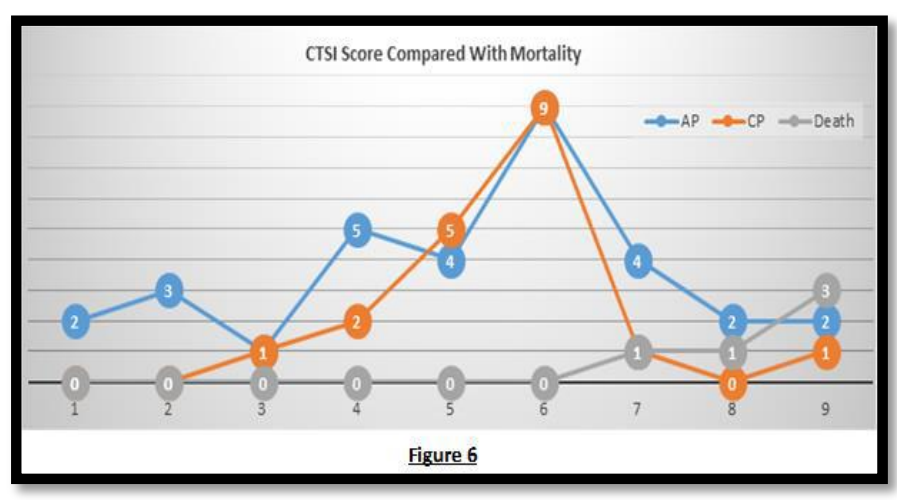

e) Treatment and Final Outcome:

The treatment administered in the cases according to their diagnosis and requirement and the final outcome of the admissions is concisely presented in table 3. $50.98 \%$ cases were managed conservatively, of which, 20 cases were of AP and the remaining six cases of CP. Of these 26 cases who were treated conservatively, 13 cases were discharged with advice of follow-up. 11 cases were discharged against medical advice, one was referred to another hospital and one case of AP died during treatment. The remaining cases were managed surgically, including endoscopic procedures. These included $12 \mathrm{AP}$ and $13 \mathrm{CP}$ cases. Of these, 20 cases were discharged with follow-up advice, two cases of $\mathrm{AP}$ and $\mathrm{CP}$ each deceased and one case of CP was discharged against medical advice before being declared fit by the doctors.

\begin{tabular}{|c|l|c|c|c|c|c|}
\hline \multirow{2}{*}{ Treatment } & Diagnosis & \multirow{2}{*}{$\begin{array}{c}\text { No. of } \\
\text { Cases }\end{array}$} & & & \multicolumn{5}{|c|}{ Outcome } \\
\cline { 5 - 8 } & & & Discharged & DAMA & $\begin{array}{c}\text { Transferred } \\
\text { out }\end{array}$ & Death \\
\hline \multirow{2}{*}{ Conservative } & AP & 20 & 11 & 7 & 1 & 1 \\
\cline { 3 - 7 } & CP & 6 & 2 & 4 & 0 & 0 \\
\hline \multirow{2}{*}{ Surgical } & AP & 12 & 9 & 1 & 0 & 2 \\
\cline { 2 - 7 } & CP & 13 & 11 & 0 & 0 & 2 \\
\hline \multicolumn{7}{|c|}{ Table 3 } \\
\hline
\end{tabular}

\section{f) Complications:}

Table 4 illustrates the various complications that were noted in the cases. $15 \mathrm{AP}$ and $12 \mathrm{CP}$ cases had complications. While pseudocyst was the most common complication in case of AP, it was also seen in a few cases of CP. The reports noted that, on further investigations, four cases of $\mathrm{CP}$ were found to have pancreatic carcinoma making it a common complication of $\mathrm{CP}$. Other complications that were observed were Diabetic Ketoacidosis (DKA), metabolic encephalopathy and metabolic encephalopathy with Acute Renal Failure (ARF) associated with AP and pancreatic duct (PD) calculi in case of CP.

\begin{tabular}{|l|l|}
\hline \multicolumn{2}{|l|}{ AP } \\
\hline Pseudocyst & 11 \\
\hline DKA & 2 \\
\hline Metabolic Encephalopathy & 1 \\
\hline $\begin{array}{l}\text { Metabolic Encephalopathy } \\
\text { with ARF }\end{array}$ & 1 \\
\hline CP & 4 \\
\hline Pancreatic Carcinoma & 2 \\
\hline Pseudocyst Table 4 & 2 \\
\hline PD Calculi & \\
\hline \multicolumn{2}{|c|}{} \\
\hline
\end{tabular}




\section{Discussion}

a) Demographic data: The average age of incidence of pancreatitis in the western countries was noted to be within the range of 35-50 years. In this study, the average age of the cases was found to be 38.39 years. The median age was 34 and 34.5 years in case of AP and CP respectively. In a similar study of acute pancreatitis from South Africa, another developing tropical country like ours, the median age of the patients was 37 years ${ }^{\text {[27] }}$. In India, a previous nationwide study of CP conducted in 2008 reported the mean age of incidence to be around 39.7 years ${ }^{[7]}$. In this study, the mean age of incidence of $\mathrm{CP}$ was found to be 38.54 years. This clearly proves that along with the rise in the incidence of pancreatitis all over the world, the average age of incidence of pancreatitis is decreasing.

Males constituted about $90.19 \%$ of the sample while females made up $9.8 \%$. This may be attributed to the pattern of prevalence of the two chief aetiological factors, namely, alcohol ${ }^{[23]}$ and tobacco consumption which is more in males as compared to females. Besides, in the rural Indian population, males tend to be more cared for; medical intervention is sought early for the males denying the female population the basic medical help. Such factors may be responsible for the skewed sex ratio found in this study.

The sample under study comprised of typical rural population who were either illiterate, or had hardly completed their basic primary/secondary schooling. The cases belonged to lower or lowermiddle class of the recently modified BG Prasad classification ${ }^{[28]}$.

b) Aetiological factors: Typical rural diet in Maharashtra was found to be relatively rich in carbohydrates but deficient in fat and protein content. This finding was found to be in contrast to that of Sarles et al. ${ }^{[17]}$ who documented the corelation of a diet containing a high fat and protein content and pancreatitis. However, the finding of this study is in accordance with a study of tropical pancreatitis from India, in which, the cases had a similar carbohydrate rich and protein, fat deficient $\operatorname{diet}^{[19]}$.

Various addictions like alcohol consumption, tobacco chewing, beedi and cigarette smoking were present in the cases under study. In this study, alcohol consumption, present in $49.01 \%$ $(n=25)$ cases, was found to be the leading cause of pancreatitis.

These alcohol consumers, all males, were found to be consuming alcohol for a number of years ranging from five years to 30 years. $92 \%(n=23)$ of these alcohol consumers were diagnosed to be suffering from AP and the remaining $8 \%(n=2)$ from CP. The age from which the alcohol consumption started was from as low as 15 years. Studies have reported that there is rise in per capita consumption of alcohol in India[29] with a higher prevalence in the lesser educated and poor $[23,30]$

As seen in figure 7 , the maximum number of alcohol consumers were present in the age group 31-40 years closely followed by the age group 21 30 years. As we proceed up the age group, the number of alcohol consumers decreased. From figure 8 , which explains the relation of total years of alcohol consumption to the diagnosis, it is evident that alcohol consumption led more towards AP rather than $\mathrm{CP}$.

Tobacco consumption, making the second largest contribution to the aetiology of pancreatitis, was present in two chief forms; viz., tobacco chewing and smoking (beedis or cigarettes). Some cases were associated with combined consumption of tobacco and alcohol. While only four cases were found to be smokers, three of whom were diagnosed with AP, 16 cases were tobacco chewers, of which, 12 were cases of AP with duration of consumption ranging from three years to 25 years. The remaining four cases of tobacco chewers were cases of $\mathrm{CP}$ who had been consuming tobacco from 10 to 40 years. Of these, three cases, who had been consuming tobacco from 20 to 40 years, had the complication of pancreatic carcinoma, none of which, incidentally, had ever consumed alcohol. Studies have shown 
that though combined alcohol and tobacco consumption greatly increases the risk of pancreatitis, tobacco consumption is an independent and significant risk factor for $\mathrm{CP}$ ${ }^{[16,58]}$. The available western literature concerning tobacco consumption is with regards to cigarette smoking only. Effects of beedi smoking and tobacco chewing, common habits in India, need to be further studied.

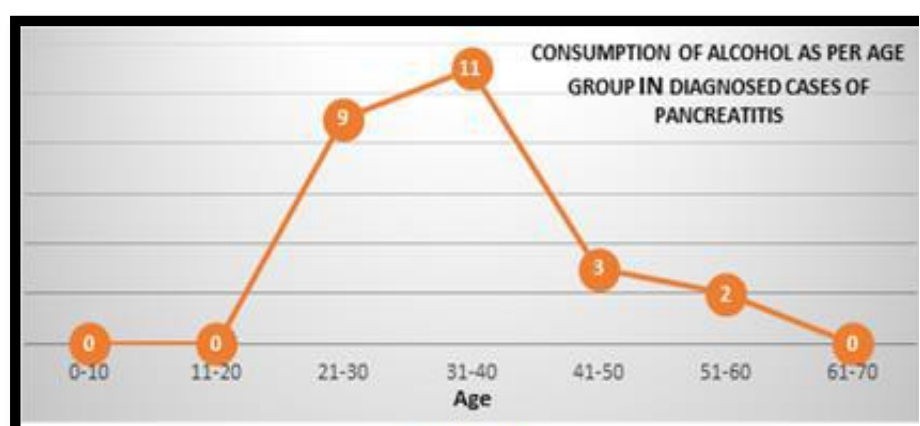

Figure 7

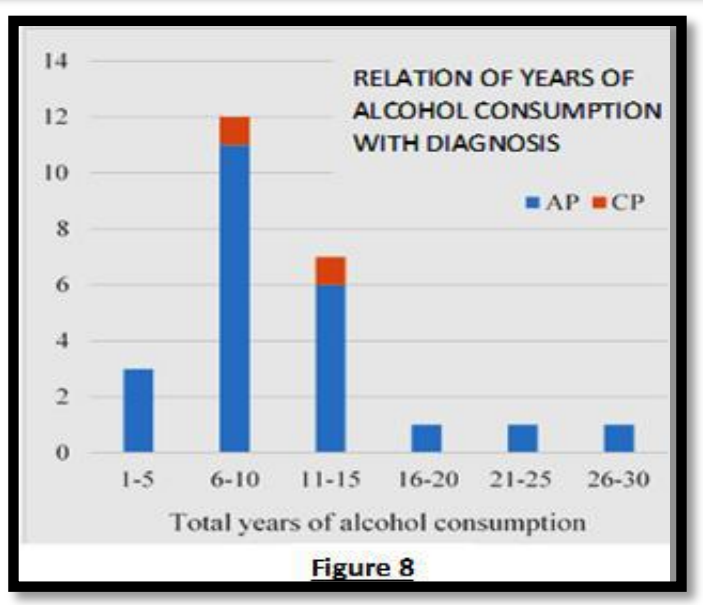

The contribution of gallstones to the aetiological factors of pancreatitis, though quite big, is lesser than that of alcohol and tobacco abuse. The present literature states that gallstones are the most common cause of AP and more so in females consistent with the demographic distribution of gallstones ${ }^{[1,12,20]}$. Accordingly, all the female cases in this study were found to be suffering from gallstone linked pancreatitis. However, in this study, it was found that the contribution of gallstones was third, next to alcohol and tobacco and they led more to $\mathrm{CP}$ rather than AP. These results are in confirmation with those of $\mathrm{D}$ Premkumar who found that alcohol contributed more to the aetiology of pancreatitis than gallstones in Indian population ${ }^{[51]}$.
Contribution of the medical procedure, ERCP, commonly employed for diagnostic and therapeutic purposes in the common bile duct and the pancreatic duct pathologies was relatively huge. However, most of these cases also had gallstone pathology. So it cannot be definitely said whether ERCP was a direct cause or a contributory factor in the pathogenesis of pancreatitis. Pancreatitis has, however been an established complication of this procedure ${ }^{[32,33]}$.

A history of previous abdominal trauma or intraabdominal procedures which may cause direct or indirect injury to pancreas was present in a few cases.

However, in $11 \%$ of the cases, the pancreatitis was idiopathic. No probable cause for pancreatitis could be found in these cases. The age range of these cases was from 17 to 65 years but clustering more in the young or middle age group. The occurrence of CP was more in these cases. Such pancreatitis of unknown origin have been noted in some other studies as well ${ }^{[7,34,35]}$. These cases may have been victims of Tropical Pancreatitis [19]. However, no definite criteria is available to label a particular case as Tropical Pancreatitis.

c) Clinical features: Severe abdominal pain was found to be characteristic of pancreatitis; be it acute or chronic. A number of hospitalisations in chronic pancreatitis are owed to pain ${ }^{[36]}$. In this study, pain was found to be the most common symptom which was present in all the cases of both, acute as well as chronic pancreatitis. Though the degree of pain varied from a mild dragging discomfort to severe acute pain in upper abdomen, no definite pattern was detected. Hardly any cases described all the factors of the classical pain pattern (sudden onset, severe, agonising, - upper abdominal pain radiating to back) of acute pancreatitis. Table 5 shows the common locations of pain. Maximum number of patients $(n=27)$ gave the history of pain in abdomen (17 AP and $10 \mathrm{CP}$ ) while seven cases said the pain was in abdomen and it was radiating to back (5 AP and 2 $\mathrm{CP})$. The pain was localised to epigastrium in six 
patients (4 AP and $2 \mathrm{CP}$ ), four cases gave history of epigastric pain radiating to back ( $2 \mathrm{AP}$ and 2 $\mathrm{CP})$ while two cases said the epigastric pain was radiating to the umbilical region (both AP). One patient each came with pain localised around umbilicus (CP) and right flank pain radiating to back (AP). Three cases reported pain localised to left hypochondrium (1 AP and $2 \mathrm{CP}$ ).

\begin{tabular}{|l|l|}
\hline Location of pain & $\begin{array}{l}\text { Percenta } \\
\text { ge }\end{array}$ \\
\hline Abdomen & 52.95 \\
\hline Abdomen radiating to back & 13.72 \\
\hline Epigastrium & 11.76 \\
\hline Epigastrium radiating to back & 7.84 \\
\hline Left hypochondrium & 5.88 \\
\hline $\begin{array}{l}\text { Epigastrium radiating to umbilical } \\
\text { region }\end{array}$ & 3.92 \\
\hline Umbilical region & 1.96 \\
\hline Right flank radiating to back & 1.96 \\
\hline \multicolumn{2}{|c|}{ Table 5 } \\
\hline
\end{tabular}

Regarding the type of pain, data of only 21 cases is available. Of these, six cases gave a history of an agonising pain in the abdomen; all of which were diagnosed as cases of AP while another case of AP, though giving a history of agonising pain, said it was of pricking type. AP also presented itself as dull aching and non-specifically intermittent pain in two cases each while as a burning, intermittently throbbing, pricking and colicky pain in a case each. Of the six $\mathrm{CP}$ cases, two said the pain was a non-specific intermittent type, another two stated that the pain was of dull aching type and one case each said the pain was pricking and intermittent colicky type. Overall, AP, owing to the acute inflammatory process, had an obvious acute presentation associated more with severe pain while $\mathrm{CP}$, following a chronic inflammatory course, presented with mild to moderate, continuous pain.

Data of aggravating and relieving factors of only five and three cases respectively is available. Four cases stated that pain got aggravated after food, lying down or work in a case each and just lying down in one case. Three cases said either bending down, sitting or rest relieved the pain.

Therefore, no typical pattern of pain can be definitely associated and looked for in the diagnosis of pancreatitis; acute or chronic.
Similarly, no factors can confidently be said to aggravate or relieve the pancreatic pain. A study in USA found that the current treatment modalities for pancreatic pain do not address the 'underlying mechanisms of sensitization and amplification of the peripheral and central pain signals', thus explaining the high level of failure of medical and surgical treatment of pain due to pancreatitis ${ }^{[37]}$.

Regarding the symptoms, along with pain, history of fever, nausea, vomiting, reduced appetite, anorexia and altered bowel habits were commonly narrated by the patients. However, on records, relatively few patients were found to be febrile. This may be due to the liberal use and over the counter availability of antipyretics in every other pharmaceutical. The nausea and vomiting was present relatively more in AP as compared to $\mathrm{CP}$. This can be attributed to the raised intraabdominal pressure, inflammatory reactions and its by-products, high degree of fluid and electrolyte imbalance and severe acute pain in AP and chronic dyspepsia owing to pancreatic insufficiency and failure in case of CP. Reduction in appetite and anorexia were also commonly seen which may be the gradual result of abdominal pain, nausea, vomiting and chronic indigestion. Though literature shows that steatorrhea is pathognomonic of pancreatitis ${ }^{[38]}$, it was barely found to be prevalent in the cases of this study. This may partly be attributed to the fat deficient diet and lack of stool examination in the cases under this study. Rather, $13.72 \%$ of the cases had constipation. In fact, $74.5 \%$ of the cases gave a history of normal bowel habits, almost equally in $\mathrm{AP}$ as well as $\mathrm{CP}$. This shows that a change in bowel habits is unlikely to be a feature of pancreatitis, be it acute or chronic.

However, further stool examinations need to be conducted before declaring the absence of steatorrhea.

As far as icterus is concerned, from the results, it can be safely concluded that icterus is more likely to be associated with chronic pancreatitis or acute on chronic pancreatitis. 
On per abdomen examination, abdominal tenderness, distension, guarding, rigidity and a palpable mass were seen in both types of pancreatitis. Tenderness, in spite of it being a feature of acute inflammatory process, was equally seen in $\mathrm{CP}$ as well pointing towards the recurrent acute attacks overlapping the CP. Distension of abdomen, seen in both AP as well as $\mathrm{CP}$, may be a result of various processes like diffuse peritonitis or ascites. Thus, abdominal distension, causing pain in itself, creates further difficulty in reaching to a conclusion of cause of pain in pancreatitis. The protective mechanisms of the abdominal wall seen in events of inflammatory insults, guarding and rigidity were more specific to acute attacks. This may be because of peritonitis and paralytic ileus generally accompanying AP. A palpable mass proved to be either due to liver enlargement or pancreatic complications like oedematous, bulky pancreas, pseudocyst or pancreatic carcinoma.

$13.72 \%$ cases confirmed having diabetes mellitus, relatively more in CP than in AP. This can be explained on the basis that chronic pancreatitis results in loss of both exocrinal and endocrinal pancreatic tissue whereas, in case of AP, there is acute dysfunction due to inflammation. It is a matter of controversy whether DM is a cause or effect of pancreatitis. DM was more often seen in alcoholics than non-alcoholics. This is in confirmation with the findings of $\mathrm{V}$ Balakrishnan et al. ${ }^{[7]}$.

d) Laboratory investigations: Almost all the cases were found to be anaemic. This can be due to the poor nutritive value of the diet consumed by the rural population. Leucocytosis, being an integral feature of inflammation was seen in most of the cases. Acute inflammatory processes are characterised by neutrophilic leucocytosis while lymphocytosis is a feature of chronic inflammation. Comparatively, leucocytosis was more often seen in AP than in CP. Blood group data of 37 cases was available. In this data, the most common blood group observed was $\mathrm{B}+\mathrm{ve}$
(51.35\%), followed by O+ve (21.62\%). Other blood groups found were $\mathrm{AB}+\mathrm{ve}(13.51 \%), \mathrm{A}+\mathrm{ve}$ $(10.81 \%)$ and B-ve $(2.7 \%)$. A study found that the incidence of pancreatitis was more in non-O blood groups ${ }^{[20]}$. The blood group factor needs to be further explored with larger sample sizes.

Biochemical abnormalities encountered in the cases were raised serum amylase and lipase levels, deranged liver enzymes, RFTs, serum electrolytes and serum calcium levels. Serum amylase ranged from normal to raised levels. However, in the five cases where the serum lipase was done, it was raised to more than thrice the normal level. Serum lipase has been found to be more sensitive in the diagnosis of pancreatitis ${ }^{[39]}$. Among the liver enzymes, alkaline phosphatase was raised in $93.75 \%$ of the AP cases while only in $84.21 \%$ of the $\mathrm{CP}$ cases. However, when it comes to the hepatic intra-cellular enzymes, Aspartate Transaminase (AST) and Alanine Transaminase (ALT), they were raised more often in case of CP (63.15\%) than in AP (56.25\%). This may be the result of chronic inflammatory changes in the pancreatic parenchyma of $\mathrm{CP}$ which include fibrosis, calcifications, pancreatic duct calculi, etc. which lead to gradual stasis of secretions in the common bile duct causing retrograde injury and damage of the liver parenchyma and subsequent raise in the liver enzymes. However, the incidence of such changes in the pancreatic parenchyma is lesser in AP. In the RFTs, the rise in blood urea was found to be more than the rise in serum creatinine in both, AP and CP. This is the result of the fluid and electrolyte imbalance that occurs in pancreatitis and the associated catabolic state that is a feature of any inflammatory process resulting in a rise in the breakdown products of proteins thus raising the BUN (Blood Urea Nitrogen) levels. On the other hand, serum creatinine, which is an indicator of renal function is affected in severe cases which are associated with multiorgan failure. Pancreatitis precipitates calcium assaponified lesions in the abdomen causing hypocalcemia. In this study, it was found that hypocalcemia was associated with a poor 
prognosis. In $62.5 \%$ of the cases who showed hypocalcemia, outcome was fatal. Though serum sodium and potassium levels were deranged in most of the cases of both $\mathrm{AP}$ and $\mathrm{CP}$, the result regarding their definite rise or fall was found to be ambiguous.

Abdominal ultrasonography is the foremost investigation done in any case of abdominal pathology. The available USG reports of the cases mostly showed heterogeneous, bulky pancreas with cystic or calcific changes in cases of AP. Current gallstones were also visualised in some cases of AP. On the other hand, the changes in the pancreas of $\mathrm{CP}$, along with heterogeneity, hypoechoic, cystic and calcified areas, were dilated pancreatic duct, pancreatic duct calculi, atrophic changes and masses. However, USG has its limitations when it comes to the diagnosis of pancreatitis. Obesity, overlying small and large bowel gas interferes with proper pancreatic imaging making the interpretation of pancreas itself unsatisfactory.

The CTSI score calculated from the available CT scan reports was compared to the treatment modalities employed as per requirement; it was found that conservative treatment was sufficient in most of the cases with CTSI scores under four. However, the higher the CTSI score, more surgical interventions were found to be necessary. Also, it was noted that more the CTSI score, more were the complications and mortality rates. Thus, it confirms that CTSI scores based on CT scans are precise prognostic indicators in cases of pancreatitis ${ }^{[26]}$. CT scan has become a great tool for the diagnosis of not only pancreatic but numerous other pathologies of the body as well. In view of this, a CT scanner, if made available at the first referral unit of health care centres, early and accurate diagnosis of various ailments will be greatly facilitated.

e) Treatment and outcome: $64.70 \%$ of the cases were successively discharged with follow-up advice. But $23.52 \%(n=12)$ cases were discharged against medical advice encumbering the proper analysis of final outcome of the cases. These cases who sought DAMA were mostly middle-aged. This could be because of the fact that they, being working class population who managed on daily wage basis could not afford to miss a day's wages more than what was absolutely necessary. $9.80 \%$ of the cases culminated in death in spite of rigorous treatment. The mortality among the surgically managed cases of pancreatitis was fourfold as compared to those treated conservatively. However, surgical interventions were resorted to in the cases in which it was unequivocally essential.

f) Complications: complications were noted more in cases of CP than in AP. Pseudocyst was noted as most common complication in study, also it was observed that alcoholic group in study was more likely affected. Other complications such as renal failure, neoplasms were also noted. The presence of these complications, and the resultant morbidities, are great contributing factors to the decreased quality of life in patients suffering from pancreatitis.

\section{Conclusion}

Pancreatitis is a disease of middle age, prevalent more in males than in females. Its average age of incidence in the rural population has been found to be decreasing. Further large scale incidence studies are necessary to confirm if this pattern is seen all over India.

This study reaffirms that alcohol is the major factor associated with pancreatitis in the rural population in western India. The aetiological factors responsible for pancreatitis in decreasing order of frequency were alcohol abuse, tobacco consumption, gall stones followed by the iatrogenic cause, ERCP\& idiopathic pancreatitis. However, further exploration of the role of the various aetiological factors like diet, tobacco consumption in Indian set-up with large sample size is necessary.

At the basic health care centres in India, diagnosis of pancreatitis can be reached by a high degree of 
suspicion while approaching any patient presenting with pain in abdomen. The pain may range from severe, agonising to dull, aching pain anywhere in the upper abdomen and may or may not radiate to the back. Other findings that may aid in its diagnosis are a history of high carbohydrate, low fat and low protein diet, alcohol or tobacco abuse, gallstones, ERCP and trauma. Fever, nausea, vomiting, tenderness and distension of abdomen

are generally present pointing more towards AP than CP. A palpable mass in abdomen should be investigated further with a suspicion of $\mathrm{CP}$. The presence of anorexia, steatorrhea is generally not definite in Indian patients. A preference to serum lipase over serum amylase is more specific leaving no space for doubt in diagnosing pancreatitis. Accompanying leucocytosis, raised liver enzymes, blood urea, and decreased serum calcium levels strongly point towards pancreatitis. The diagnosis can further be confirmed by CT scan which also helps in evaluating the severity and prognosis of the cases. These findings form sufficient diagnostic criteria for the suspicion and diagnosis of pancreatitis in Indian scenario.

Mild cases of pancreatitis can be managed conservatively, but with increasing severity based on CTSI Score surgical interventions are necessitated. In line with this, conservatively managed cases had better outcome than the surgical modality of treatment.

Though most of the findings reported in this present study are in conformity with the studies of past, for certain variables, a variance of association of epidemiological and clinical factors in pathogenesis, occurrence, clinical profile and outcome of pancreatitis was noted. This study comprised of a relatively small sample size which is a great limitation for arriving at definite conclusions; for this, further studies are required both epidemiological and clinical.

Through large scale prospective studies, ensuring comprehensive data collection, the observations of the epidemiology, symptoms and signs with the supportive investigations aiding the diagnosis of pancreatitis presented in this study may be confirmed and evolved into a clinico-pathological score for diagnosis and management of pancreatitis in Indian scenario.

\section{Acknowledgements}

Dr. Jayant Gadekar ${ }^{1}$, Dr. Mrs. Zambare ${ }^{2}$

${ }^{1}$ Professor \& Head of the Department of General Surgery.

${ }^{2}$ Professor \& Head of the Department of Preventive and Social Medicine.

Dr. Vitthalrao Vikhe Patil Foundation's Medical College \& Hospital, Near Govt. Milk Dairy, ViladGhat, Ahmednagar, Maharashtra, India.

\section{References}

1. Peter Lee, Tyler Stevens; Acute Pancreatitis. Cleveland Clinic 4th Annual Gastroenterology \& Hepatology Symposium, February 2014.

2. Lankisch PG, Banks PA. Pancreatitis. New York: Springer; 1998.

3. Ministerodel Lavoro, della Salute e delle Politiche Sociali. Available online: http://www.ministerosalute.it/programmaz ione/sdo/ric_informazioni/sceltadia.jsp (accessed 14 July 2009).

4. Garg PK, TondonRK; Survey on chronic pancreatitis in the Asia-Pacific region. $J$ Gastroenterol Hepatol 2004; 19:998-1004.

5. Moynihan B. Acute pancreatitis. Ann Surg 1925;81:132-42.

6. Raffaele Pezzilli, Antonio M. MorselliLabate; Alcoholic Pancrea-titis: Pathogenesis, Incidence and Treatment with Special Reference to the Associated Pain. Int. J. Environ. Res. Public Health 2009, 6, 2763-2782; doi:10.3390/ijerph6112763.

7. Vallath Balakrishnan et al; Chronic Pancreatitis: A Prospective Nationwide Study of 1,086 Subjects from India.JOP. J Pancreas 2008;9(5):593-600.

8. FrossardJL, Steer ML, Pastor CM. Acute pancreatitis. Lancet. 2008; 371:143-52. PMID 18191686. \{PubMed: 18191686\}

9. GoldacreMJ, Roberts SE. Hospital admission for acute pancreatitis in an 
English population, 1963-98: database study of incidence and mortality. BMJ. 2004; 328:1466-9. PMID 15205290. \{PubMed: 15205290\} .

10. O'Farrell A, Allwright S, Toomey D, Bedford D, Conlon K. Hospital admission for acute pancreatitis in the Irish population, 1997 2004: could the increase be due to an increase in alcohol related pancreatitis? J Public Health (Oxf). 2007; 29:398-404. PMID 17998260. \{PubMed: $17998260\}$

11. Spanier BW, Dijkgraaf MG, Bruno MJ. Trends and forecasts of hospital admissions for acute andchronic pancreatitis in the Netherlands. Eur J GastroenterolHepatol. 2008; 20(7):653-8. PMID18679068. \{PubMed: 18679068\}

12. Yadav D, Lowenfels AB. Trends in the epidemiology of the first attack of acute pancreatitis: asystematic review. Pancreas. 2006; 33:323-30. PMID 17079934. \{PubMed: 17079934\}

13. Gullo L; Alcohol and chronic pancreatitis: leading or secondary etiopathogenic role? JOP. J Pancreas 2005:6:68-72 PMID15650289.

14. Hyacinth M Irving, Andriy V Samokhvalov, JurgenRehm; Alcohol as a risk factor for pancreatitis: A systemic review and meta-analysis. Author manuscript; PMC 2012 March 12 JOP.;10(4):387-392.

15. Stella Yen, Chung-Cheng Hsieh, Brian MacMahon; Consumption of alcohol and tobacco and other risk factors for pancreatitis. Am. J. Epidemiol. (1982)116(3):407-414.

16. Lin, Yingsong et al; Cigarette smoking as a risk factor for chronic pancreatitis: A case-control study in Japan. Pancreas Aug, 2000 Vol.21-Issue 2: pp109-114.

17. Sarles H.; An international survey on nutrition \& pancreatitis. Digestion 1973;9:389-403.
18. HariharanRegunath et al; Anthropometric measurements of nutritional status in chronic pancreatitis in India: comparison of tropical and alcoholic pancreatitis. Indian J Gastroenterol (March-April 2011)30(2):78-83DOI $\quad 10.1007 / \mathrm{s} 12664-$ 011-0093-9.

19. V Balakrishnan et al; Tropical pancreatitis -a distinct entity, or merely a type of chronic pancreatitis? Indian Journal of Gastroenterology 2006Vol.25 March April.

20. Dhiraj Yadav, AB Lowenfels. The epidemicology of pancreatitis and pancreatic cancer. Gastroenterology. 2013 June; 144(6): 1252-1261. doi:10.1053/j.gastro.2013.01.068.

21. Verlaan M et al; Genetic polymorphisms in alcohol metabolizing enzymes and chronic pancreatitis. Alcohol Alcohol2004;39:20-4.

22. Maruyama et al.; Autoimmune pancreatitis can develop into chronic pancreatitis. Orphanet Journal of Rare Diseases 2014,9:77.

23. Das, S.K. et al; Alcohol: its health and social impact in India. Natl. Med. J. India 2006,1994-99.

24. Talamini G, Falconi M, Bassi C, Sartori N, Salvia R, Caldiron E, et al. Incidence of cancer in the course of chronic pancreatitis. Am J Gastroenterol 1999; 94:1255-60.

25. Lowenfels AB, Maisoneuve $P$, Cavallini G, AmmannRW, Lankisch PG, Anderson JR, et al. Pancreatitis and the risk of pancreatic cancer. N Engl J Med 1993; 328:1433-7.

26. Balthazar EJ, Robinson DL, MegibowAJ, RansonJHC. Acute pancreatitis: value of CT in establishing prognosis. Radiology 1990;174:331-6.

27. F. Anderson et al. Acute pancreatitis: Demographics, aetiological factors and outcomes in a regional hospital in South 
Africa. SAJS Vol 46, No. 3, August 2008: 83-86.

28. Mangal A, Kumar V, Panesar S, Talwar R, Raut D, Singh S. Updated BG Prasad socioeconomic classification, 2014: A commentary. Indian $\mathbf{J}$ Public Health 2015;59:42-4.

29. Rajendran SD (ed). Globalization and increasing trend of alcoholism. Community Health Cell, for the Asia Social Forum, 2-7 January 2003, Hyderabad, India.

30. Chakravarthy C. Community workers estimate of drinking and alcohol-related problems in rural areas. Indian J Psychol Med 1999;13:49-56.

31. M Bourliere, M Barthet, P Berthezene, J P Durbec, $\mathrm{H}$ Sarles. Is tobacco a risk factor for chronic pancreatitis and alcoholic cirrhosis? Gut, 1991, 32, 1392-1395.

32. D Premkumar. Etiological factors affecting the clinical features, prognosis and management of acute pancreatitis. ActaMedicaScientia $02 \quad\{01\} \quad$ (2015) www.asdpub/index.php/ams

33. Paul Georg Lankisch, MinotiApte, Peter A Banks. Acute Pancreatitis. Published Online January 21, 2015 http://dx.doi.org/10.1016/S01406736(14)60649-8.

34. Frulloni, L.; Gabbrielli, A.; Pezzilli, R.; Zerbi, A.; Cavestro, G.M.; Marotta, F.; Falconi, M.; Gaia, E.; Uomo, G.; Maringhini, A.; Mutignani, M.; Maisonneuve, P.; di Carlo, V.; Cavallini, G. Chronic pancreatitis: report from a multicenter Italian survey (Pan CroInf AISP) on 893 patients. Dig. Liver Dis. 2009, 41, 311-317.

35. Montalto, G.; Carroccio, A.; Soresi, M.; Ficano, L.; Notarbartolo, A. Chronic pancreatitis in Sicily. Preliminary reports. Ital. J. Gastroenterol. 1990, 22, 33-35.
36. Mullady DK, Yadav D, Amann ST, O'Connell MR, Barmada MM, EltaGH, et al. Type of pain, painassociated complications, quality of life, disability and resource utilisation in chronic pancreatitis: aprospective cohort study. Gut 60(1):77-84, 2011. PMID: 21148579.

37. D Dominguez, K Kirkwood. Pathogenesis of pain in chronic pancreatitis. The Pancreapedia. Version 1.0, May 9, 2015.

38. DiMagno EP. A short, eclectic history of exocrine pancreatic insufficiency and chronic pancreatitis. Gastroenterology 1993; 104:1255-62.

39. S Hofmeyr, BL Warren. Serum lipase should be the laboratory test of choice for suspected acute pancreatitis. S Afr J Surg 2014;52(3):72-75. DOI:10.7196/SAJS.2003. 